\title{
Notes on Automorphic Functions: An Entire Automorphic Form of Positive Dimension is Zero*
}

\author{
Marvin I. Knopp** \\ (August 14, 1967)
}

\begin{abstract}
Several new proofs are given of the fact that an entire automorphic form of positive dimension is zero. The first proof is modeled on the method used by Hecke to estimate the Fourier coefficients of cusp forms of negative dimension. The other proofs involve well-known theorems of complete function theory.
\end{abstract}

Key Words: Automorphic forms, discontinuous groups, Fourier expansion, $H$-groups.

1. It is a result familiar in the theory of automorphic forms that an entire automorphic form of positive dimension on an $\mathrm{H}$-group is identically zero (see sec. 2 for the definitions). This follows immediately, for example, from the well-known exact formula for the Fourier coefficients of automorphic forms of positive dimension ([1], p. 314). ${ }^{1}$ Another proof is by means of a formula for the number of zeros minus the number of poles of an automorphic form in a fundamental domain. This formula (obtained by contour integration around the fundamental domain) shows that when the dimension of the form is positive, this difference is negative, and hence such a form must have poles.

In section 3 of this note we give what appears to be a new proof of this result by using the method Hecke employed to estimate the Fourier coefficients of cusp forms of negative dimension ([1], p. 281). ${ }^{1}$ This proof is simpler and more direct than the proofs mentioned above. In sections 4-5 we give two variations of this method. The method of section 5 is applicable to a larger class of groups than the $H$-groups, and in particular applies to compact groups and groups conjugate to $H$-groups.

2. A group $\Gamma$ of real linear fractional transformations acting on $\mathscr{H}$, the upper half-plane $\operatorname{Im} \tau>0$, is an $H$-group provided

(i) $\Gamma$ is discontinuous on $\mathscr{H}$, but is not discontinuous at any point of the real line,

(ii) $\Gamma$ is finitely generated, and

(iii) $\Gamma$ contains translations.

With each transformation $V \epsilon \Gamma$ we associate a real $2 \times 2$ matrix $\left(\begin{array}{ll}a & b \\ c & d\end{array}\right)$ such that

\footnotetext{
*An invited paper.

**Present address: The University of Wisconsin, Madison, Wisc. 53706.

${ }^{1}$ J. Lehner, Discontinuous groups and automorphic functions, Math. Surveys No. 8, Amer. Math. Soc., Providence, R.I., 1964.
}

$$
V \tau=\frac{a \tau+b}{c \tau+d}, \quad \tau \epsilon \mathscr{H},
$$

and $a d-b c=1$. This can be done in precisely two ways since $\pm\left(\begin{array}{ll}a & b \\ c & d\end{array}\right)$ are both associated with $V$.

Since $\Gamma$ is finitely generated it has finitely many parabolic cusps in any fundamental region $R$, and since $\Gamma$ contains translations $R$ has at least one such cusp. Suppose $R$ is a fundamental region for $\Gamma$ with parabolic cusps $P_{1}, \ldots ., P_{\mu}$. Without loss of generality we may assume no $P_{i}=\infty$. For if say $P_{1}=\infty$, let $A \epsilon \Gamma$ be such that $A$ does not fix $\infty$, that is $A$ is not a translation. (If every element of $\Gamma$ were a translation, then in fact $\Gamma$ would be a cyclic group and would be discontinuous at every point of the real line, contrary to assumption.) Let

$$
N=\{\tau \in \mathscr{H} \mid \operatorname{Im} \tau>1\}, \quad R_{\infty}=R \cap N .
$$

Then $R^{*}=\left(R-R_{\infty}\right) \cup A\left(R_{\infty}\right)$ is a fundamental region for $\Gamma$ in which $\infty$ is not a parabolic cusp.

Let $r$ be a real number. We want to consider functions $F(\tau)$, meromorphic in $\mathscr{H}$, such that

$$
F(V \tau)=v(V)(c \tau+d)^{-r}(F \tau),
$$

for every $V=\left(\begin{array}{ll}a & b \\ c & d\end{array}\right) \epsilon \Gamma$ and $\tau \epsilon \mathscr{H}$. Here $v(V)$ is a complex number independent of $\tau$ such that $|v(V)|=1$ for all $V \epsilon \Gamma$. In order to fix the branch of $(c \tau+d)^{-r}$ when $r$ is not an integer, for any complex number $z$ and real number $s$, we define

$$
z^{s}=|z|^{s} \cdot \exp (i \cdot s \arg z), \quad-\pi \leqslant \arg z<\pi .
$$

For each $j, 1 \leqslant j \leqslant \mu$ the stabilizer of $P_{j}$ in $\Gamma$ (that is 
$\left.\left\{M \epsilon \Gamma \mid M P_{j}=P_{j}\right\}\right)$ is a cyclic group generated by a parabolic element of $\Gamma$. From this fact, eq (1), and the fact that $F(\tau)$ is a meromorphic in $\mathscr{H}$, it can be deduced $\left(\left[1\right.\right.$, p. 157]) that $F(\tau)$ has an expansion "at $P_{j}$ " of the form

$$
F(\tau)=\left(\tau-P_{j}\right)^{r} \sum_{n=-\infty}^{\infty} b_{n}(j) e^{2 \pi i\left(n+k_{j}\right) A_{j}^{-1} \tau / \lambda_{j}}, \quad 1 \leqslant j \leqslant \mu,
$$

where $A_{j}$ is a real linear fractional transformation such that $A_{j}(\infty)=P_{j}, 0 \leqslant k_{j}<1$, and $\lambda_{j}>0$.

Suppose that $\dot{F}(\tau)$ is meromorphic in $\mathscr{H}$, satisfies (1), and for each $j$ only finitely many terms such that $n<0$ appear in (2). Then we say that $F(\tau)$ is an automorphic form of dimension $r$ with respect to the group $\Gamma$ and the multiplier system $v$. If $F(\tau)$ is holomorphic in $\mathscr{H}$ and for each $j$ only terms such that $n+k_{j} \geqslant 0$ appear in (2) we say that $F(\tau)$ is an entire automorphic form. If $F(\tau)$ is an entire form such that only terms with $n+k_{j}>0$ appear in (2), we say that $F(\tau)$ is a cusp form.

3. We now assume that $F(\tau)$ is an entire automorphic form of dimension $r>0$ with respect to $\Gamma$. We will show that

$$
|F(\tau)|=|F(x+i y)| \leqslant K y^{r / 2}
$$

for all $\tau \epsilon \mathscr{H}$, where $K$ is independent of $x$ and $y$. Consider the function

$$
\varphi(\tau)=\varphi(x+i y)=y^{-r / 2}|F(x+i y)|, \quad \tau \epsilon H .
$$

A simple calculation shows that for $\tau=x+i y$,

$$
\operatorname{Im} V \tau=\frac{\operatorname{Im} \tau}{|c \tau+d|^{2}}=\frac{y}{|c \tau+d|^{2}},
$$

for $V=\left(\begin{array}{ll}* & * \\ c & d\end{array}\right) \epsilon \Gamma$. This fact and (1) together imply that $\varphi(V \tau)=\varphi(\tau)$ for all $V \epsilon \Gamma$.

We will show next that as $\tau \rightarrow P_{j}$ from within $R^{*}$, $\varphi(\tau) \rightarrow 0(1 \leqslant j \leqslant \mu)$. Now $\tau \rightarrow \infty$ from within a vertical strip if and only if $A_{j} \tau \rightarrow P_{j}$ from within $R^{*}$. Consider

$$
\begin{aligned}
\varphi\left(A_{j} \tau\right) & =\left(\operatorname{Im} A_{j} \tau\right)^{-r / 2}\left|F\left(A_{j} \tau\right)\right| \\
& =\frac{y^{-r / 2}}{|c \tau+d|^{-r}}\left|A_{j} \tau-P_{j}\right| r\left|\sum_{n+k_{j} \geqslant 0} b_{n}(j) e^{2 \pi i\left(n+k_{j}\right) \tau / \lambda_{j}}\right|,
\end{aligned}
$$

where $A_{j}=\left(\begin{array}{ll}a & b \\ c & d\end{array}\right)$. Here we have used the expansion (2) and the fact that $F(\tau)$ is an entire form. As $\tau \rightarrow \infty$ from within a vertical strip the infinite sum is bounded. On the other hand

$$
A_{j} \tau-P_{j}=\frac{a \tau+b}{c \tau+d}-\frac{a}{c}=-\frac{1}{c(c \tau+d)} .
$$

Hence

$$
\begin{array}{r}
y^{-r / 2}|c \tau+d|^{r}\left|A_{j} \tau-P_{j}\right|^{r}=y^{-r / 2}|c \tau+d|^{r} \mid \\
\left.c(c \tau+d)\right|^{-r}=|c|^{-r} y^{-r / 2} .
\end{array}
$$

Since $r>0$, we conclude that $\varphi\left(A_{j} \tau\right) \rightarrow 0$ as $y \rightarrow+\infty$; hence $\varphi(\tau) \rightarrow 0$ as $\tau \rightarrow P_{j}$ from within $R^{*}$.

An $H$-group has a fundamental region $R$ with the property that the closure of $R$ intersects the real line only in parabolic cusps $[1$, p. 145, Th. 6G]. The fundamental region $R^{*}$ constructed in section 2 from $R$ also has this property. Therefore $\sup \left\{\varphi(\tau) \mid \tau \in R^{*}\right\}$ is actually achieved at a point of $\mathscr{H}$, since $\varphi(\tau)$ is continuous in $\mathscr{H}$. Hence there exists $K>0$ such that $\varphi(\tau) \leqslant K$ for all $\tau \in R^{*}$. Since $\varphi(V \tau)=\varphi(\tau)$ for $V \epsilon \Gamma$, $\varphi(\tau) \leqslant K$ for all $\tau \epsilon H$, and (3) follows.

We proceed to show that $F(\tau)$ is identically zero. Since $F(\tau)$ is an entire form and $\Gamma$ has translations, $F(\tau)$ has an expansion "at $\infty$ " of the form

$$
F(\tau)=\sum_{n+k \geqslant 0} a_{n} e^{2 \pi i(n+k) \tau / \lambda},
$$

where $0 \leqslant k<1$, and $\lambda>0$. This expansion is valid in all of $\mathscr{H}$. There are several ways to complete the proof. The first of these closely follows Hecke's method for estimating the coefficients of cusp forms of negative dimension.

A calculation involving (4) shows that

$$
a_{n}=\lambda^{-1} \int_{\tau_{0}}^{\tau_{0}+\lambda} F(\zeta) e^{-2 \pi i(n+k) \zeta / \lambda} d \zeta, \quad n \geqslant 0
$$

where $\tau_{0}=x_{0}+i y_{0} \epsilon \mathscr{H}$ and the integral is taken along the horizontal path. Hence by (3),

$$
\begin{aligned}
\left|a_{n}\right| & \leqslant \lambda^{-1} \cdot K y_{0}^{r / 2} \cdot \lambda e^{2 \pi(n+k) y_{0} / \lambda} \\
& =K e^{2 \pi(n+k) y_{0} / \lambda} y_{0}^{r / 2} .
\end{aligned}
$$

Letting $y_{0} \rightarrow 0+$ we get $a_{n}=0$ for $n=0,1,2, \ldots$, and therefore $F(\tau)=0$ for all $\tau \epsilon \mathscr{H}$.

4. Here is another method. There exists a linear fractional transformation $L$ which maps the open unit disk $D$ onto $\mathscr{H}$, the unit circle $T$ onto the real line (including $\infty$ ) and the complement of $\bar{D}$ onto the lower half-plane. $L$ can be chosen so that $L(1)=\infty$. Consider the function

$$
f(z)=F(L(z)), \quad z \in D .
$$

Then $f$ is holomorphic in $D$. Since $r>0$ the inequality (3) shows that $F(\tau)$ has radial limit 0 at every point of the real line, except possibly $\infty$. By (4) $F(\tau)$ is bounded uniformly for $\operatorname{Im} \tau>1$, say $|F(\tau)| \leqslant K_{1}$. On the other hand (3) implies that for $0<\operatorname{Im} \tau<1$, $|F(\tau)| \leqslant K$. Hence for $\tau \epsilon H,|F(\tau)| \leqslant \max \left(K, K_{1}\right)$. It follows that $f(z)$ is a bounded holomorphic function on $D$ with radial limit 0 at every point of $T$, except possibly at $z=1$. By a standard theorem of function 
theory ([2] pp. 300-301) $)^{2} f(z) \equiv 0$ in $D$ and therefore $F(\tau) \equiv 0$ in $\mathscr{H}$.

5. We give a slight variation of the second method which works for "compact groups" as well as $H$-groups. A discontinuous group $\Gamma$ acting on $\mathscr{H}$ is called compact if $\Gamma$ has a fundamental region $R$ whose closure is a compact subset of $\mathscr{H}$. The nomenclature stems from the fact that this condition on the group is equivalent to the compactness of the Riemann surface $\mathscr{H} / \Gamma$.

We have already proved (3) for $H$-groups. For compact groups (3) is trivial. As before, $\varphi(\tau)=y^{-r / 2}|F(\tau)|$ is invariant under $\Gamma$; also $\varphi(\tau) \leqslant K$ for $\tau \epsilon \bar{R}$, since $\varphi$ is continuous in $\mathscr{H}$ and $\bar{R}$ is a compact subset of $\mathscr{H}$. On the other hand (3) and the Schwarz reflection principle imply that $F(\tau)$ can be extended to an entire function which is zero on the real axis. Hence $F(\tau) \equiv 0$.

6 . The method of section 5 is not restricted to

${ }^{2}$ W. Rudin, Real and complex analysis (McGraw-Hill Book Co., New York, N.Y., 1966).
$H$-groups and compact groups. It can in fact be adapted without difficulty to the class of discontinuous function groups $\Gamma$ with domain of existence $\mathscr{D}$ satisfying

Condition A. $\Gamma$ has a fundamental region $R \subset \mathscr{D}$ with the property that $R \cap B d \mathscr{D}$ either is empty or consists entirely of parabolic vertices.

The method of section 5 yields the following

THEOREM. Let $\Gamma$ be a discontinous function group with a simply connected domain of existence $\mathscr{D}$ satisfying Condition A. If $\mathrm{F}(\tau)$ is an entire automorphic form of positive dimension on $\Gamma$, then $\mathrm{F} \equiv 0$ in $\mathscr{D}$.

To prove this we consider $\lambda(\tau)^{-r / 2} \cdot F(\tau)$ in place of $y^{-r / 2} \cdot F(\tau)$, where $\lambda(\tau)^{-1}$ is the Poincare metric for the hyperbolic geometry in $\mathscr{D}$. For example if $\mathscr{D}$ is the unit disk, $\lambda(\tau)=1-|\tau|^{2}$.

Condition $A$ is trivially satisfied for compact groups; for $H$-groups and their conjugates it is satisfied by ([1], p. 145, Th. 6G). By ([1], p. 133, Th. 4J) any group satisfying Condition $A$ is finitely generated.

(Paper 71B4-239) 